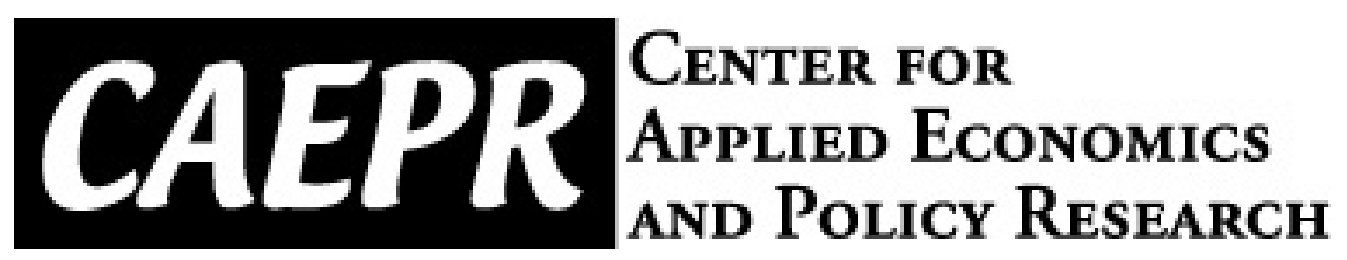

CAEPR Working Paper

\#005-2009

\title{
Club Networks with Multiple Club Memberships and Noncooperative Stability
}

\author{
Frank H. Page, Jr., Myrna H. Wooders \\ Indiana University and Vanderbilt University
}

February 24, 2009

This paper can be downloaded without charge from the Social Science Research Network electronic library at: http://ssrn.com/abstract=1348634 .

The Center for Applied Economics and Policy Research resides in the Department of Economics at Indiana University Bloomington. CAEPR can be found on the Internet at:

http://www.indiana.edu/ caepr. CAEPR can be reached via email at caepr@indiana.edu or via phone at 812-855-4050.

(C)2008 by NAME. All rights reserved. Short sections of text, not to exceed two paragraphs, may be quoted without explicit permission provided that full credit, including $₫$ notice, is given to the source. 


\title{
Club Networks with Multiple Memberships and Noncooperative Stability
}

\author{
Frank H. Page, Jr.* \\ Department of Economics \\ Indiana University \\ Bloomington, IN 47405 \\ fpage@indiana.edu \\ Phone: (812) 855-4764 \\ FAX: (812) 855-3736
}

\author{
Myrna Wooders ${ }^{\dagger}$ \\ Department of Economics \\ Vanderbilt University \\ Nashville, TN 37235 \\ m.wooders@vanderbilt.edu \\ Phone: (615) 343-0461 \\ FAX: (615) 343-8494
}

June, 2007

Current Version, February, 2009

\begin{abstract}
Modeling club structures as bipartite directed networks, we formulate the problem of club formation as a noncooperative game of network formation and identify conditions on network formation rules and players' network payoffs sufficient to guarantee that the game has a potential function. Our sufficient conditions on network formation rules require that each player be choose freely and unilaterally those clubs he joins and also his activities within these clubs (subject to his set of feasible actions). We refer to our conditions on rules as noncooperative free mobility. We also require that players' payoffs be additively separable in player-specific payoffs and externalities (additive separability) and that payoff externalities - a function of club membership, club activities, and crowding be identical across players (externality homogeneity). We then show that under these conditions, the noncooperative game of club network formation is a potential game over directed club networks and we discuss the implications of this result.
\end{abstract}

\footnotetext{
*Also, Centre d'Economie de la Sorbonne, Universite Paris 1, Pantheon-Sorbonne.

${ }^{\dagger}$ Also, Department of Economics, University of Warwick, Coventry CV4 7AL, UK.

${ }^{\ddagger}$ This paper was begun while Page and Wooders were visiting CERMSEM at the University of Paris 1 in June 2007. The authors thank CERMSEM and Paris 1 for their hospitality. The authors are especially grateful to two referees whose careful reading and thoughtful comments lead to many improvements in our paper. The authors also thank participants in the International Conference on the Formation of Social Networks, Paris, Carre des Sciences, June 28-29, 2007, especially Matt Jackson and Francis Bloch. Finally, the authors thank seminar participants at Arizona State, Arizona, Warwick,Vigo, Paris 1, Ohio State, and the Autònoma (Barcelona) for many helpful comments. (mcmnet29rf.tex) KEYWORDS: clubs, network formation games, potential games, path dominance core, Nash club equilibria, admissible set. JEL Classification Numbers: A14, D20, J00.
} 


\section{Introduction}

Club theory and the theory of local public good provision has a long history in economics, dating back to seminal papers of Charles Tiebout (1956) and James Buchanan (1965). Three types of approaches have been applied: price taking equilibrium theory; cooperative game theory, and; non-cooperative models of club/jurisdiction formation. There has been very little study, however, of club models where players can belong to multiple clubs. Also, even in situations allowing multiple memberships in clubs, no account is taken of the fact that individuals may be connected in different ways to the same club and have different connections with different clubs. Networks appear to provide a promising approach to modeling strategic club formation where players can have multiple club memberships with different connections within clubs and across clubs.

Modeling club structures as bipartite directed networks, we formulate the problem of club formation as a noncooperative game of network formation and identify conditions on network formation rules and players' network payoffs sufficient to guarantee that the game has a potential function. Our sufficient conditions on network formation rules require that each player be choose freely and unilaterally those clubs he joins and also his activities within these clubs (subject to his set of feasible actions). We refer to our conditions on rules as noncooperative free mobility. We also require that players' payoffs be additively separable in player-specific payoffs and externalities (additive separability) and that payoff externalities - a function of club membership, club activities, and crowding - be identical across players (externality homogeneity). We then show that under these conditions, the noncooperative game of club network formation is a potential game over directed club networks and we discuss the implications of this result.

The feature of free and unilateral choice of club membership in our model also appears in other non-cooperative models of club/jurisdiction formation; see, for example, Demange $(1994,2004)$ and Konishi, Le Breton and Weber $(1997,1998)$. The models and results of these papers have inspired our work. In particular, Konishi, Le Breton and Weber (1998) show that their game has a potential function, Our model differs most substantially in two important respects: players may engage in activities within multiple clubs and engage in different activities in different clubs ${ }^{1}$ and; there may be externalities between clubs - the activities of one player in a club may affect the payoffs to players in another club. Nevertheless, we are able to demonstrate that network formation games satisfying our conditions are potential games.

Our research is also related to that of Kalai and Schmeidler (1977) who introduce the concept of the "admissible set". ${ }^{2}$ To define their admissible set, take as given a set of feasible alternatives, denoted by $S$, a dominance relation $M$ and the transitive closure of $M$, denoted by $\widehat{M}$. The admissible set is the set $A(S, M)=\{x \in S$ : $y \in S$ and $y \widehat{M} x$ imply $x \widehat{M} y\}$. The admissible set describes those outcomes that

\footnotetext{
${ }^{1}$ Allouch and Wooders (2007) present a discussion of this branch of the literature, primarily concerned with nonemptiness of cores, existence of equilibrium, and core-equilibrium equivalence.

${ }^{2}$ See also Kalai, Pazner and Schmeidler (1976).
} 
are likely to be reached by any dynamic process that respects preferences. The admissible set concept can be applied to a host of game-theoretic situations, ranging from non-cooperative games, where a coalition consists of an individual player, to fully cooperative games, where any coalition can be allowed to form. As shown by Kalai and Schmeidler through a series of examples, the relationship of the admissible set to the set of Nash equilibrium depends on the definition of the dominance relation and, in some situations, the set of Nash equilibrium and the admissible set coincide. It is interesting to note that if the dominance relation is defined based on a notion of "possible replies", which can be thought of as "improving replies" (rather than best replies in the usual sense), then the admissible set is equivalent to the set of Nash equilibrium. The admissible set is related to "basins of attraction" for network formation games (Page and Wooders 2005, 2008). In the framework of the current paper, in part because of the finiteness of the strategy sets, each Nash equilibrium strategy profile is a basin of attraction and the union of all basins of attraction coincides with (the network rendition of) the admissible set. Our current paper demonstrates a class of situations where there exists singleton basins of attraction, what appears to be an important property.

\section{Club Networks with Multiple Memberships}

We begin by introducing the notion of a club network where players can have multiple club memberships. Using bipartite networks we are able to represent any such club structure in a compact and precise way.

Let $D$ be a finite set of players consisting of two or more players with typical element denoted by $d$ and let $C$ be a finite set of club types (or alternatively, a set of club labels or club locations) with typical element denoted by $c$. Finally, let $A$ be a finite set of arcs (or actions) potentially available to all players. For each player $d$ and club $c$, denote by $A(d, c)$ the feasible set of actions that can be taken by player $d$ in club $c$.

Definitions 1 (Club Networks with Multiple Memberships)

(a) A club network $G$ is a nonempty subset of $A \times(D \times C)$ such that (i) for all players $d \in D$, the section of $G$ at $d$ given by

$$
G(d):=\{(a, c) \in A \times C:(a,(d, c)) \in G\}
$$

is nonempty; and (ii) for all $(a,(d, c)) \in G, a \in A(d, c)$. Let $\mathbb{K}$ denote the collection of all such club networks.

(b) A player $d$ club network $G_{d}$ is a nonempty subset of $A \times(\{d\} \times C)$ such that for all $(a,(d, c)) \in G_{d}, a \in A(d, c)$. Let $\mathbb{K}_{d}$ denote the collection of all player $d$ club networks. 
Given club network $G \in \mathbb{K},(a,(d, c)) \in G$ means that player $d$ is a member of club $c$ and takes action $a \in A(d, c)$ in club $c$. The section of $G$ at $d$ is the set of action-club pairs listing the clubs to which player $d$ belongs and the action taken by player $d$ in each of those clubs. The set

$$
G(a, c):=\{d \in D:(a,(d, c)) \in G\}
$$

(i.e., the section of $G$ at $(a, c))$ is the set of all players who, in club network $G \in \mathbb{K}$, are members of club $c$ and take action $a$ in club $c$. Thus, the cardinality of the set $G(a, c)$, denoted by $|G(a, c)|$, is the total number of players who are members in club $c$ and take action $a$ in club $c$, and the sum $\sum_{a \in A}|G(a, c)|$ is the total number of players active in club $c .^{3}$

Note that the set of club networks is given by the union of all player networks over all players. In particular, $\mathbb{K}=\cup_{d \in D} \mathbb{K}_{d}$. Also note that given any club network $G \in \mathbb{K}$

$$
\begin{gathered}
G=\cup_{d^{\prime} \in D} G_{d^{\prime}} \\
\text { where } \\
G_{d^{\prime}}=\left\{(a,(d, c)) \in G: d=d^{\prime}\right\} .
\end{gathered}
$$

In particular, any club network $G$ is given by the union $\cup_{d^{\prime} \in D} G_{d^{\prime}}$ of a collection of player club networks $\left(G_{d^{\prime}}\right)_{d^{\prime}}$, with one player club network for each player.

Example 1 (Marketing Networks as Club Networks with Multiple Memberships) Suppose there are five firms $D=\left\{d_{1}, d_{2}, d_{3}, d_{4}, d_{5}\right\}$, two markets $C=\left\{c_{1}, c_{2}\right\}$, where $c_{1}=$ New York and $c_{2}=$ Paris, and three possible product lines $A=\left\{a_{1}, a_{2}, a_{3}\right\}$. Each firms feasible product lines appear in the list below:

$$
\begin{aligned}
& A\left(d_{1}, c\right)=\left\{a_{1}, a_{3}\right\} \text { for all } c \in C, \\
& A\left(d_{2}, c\right)=\left\{a_{1}, a_{2}\right\} \text { for all } c \in C, \\
& A\left(d_{3}, c\right)=\left\{a_{2}, a_{3}\right\} \text { for all } c \in C, \\
& A\left(d_{4}, c\right)=\left\{a_{2}, a_{3}\right\} \text { for all } c \in C, \\
& A\left(d_{5}, c\right)=\left\{a_{1}, a_{3}\right\} \text { for all } c \in C .
\end{aligned}
$$

Marketing network $G^{0}$ depicted in Figure 1 represents one possible product line-

\footnotetext{
${ }^{3}$ If $G(a, c)=\emptyset$, then $|G(a, c)|=0$.
} 
market profile for firms $D$.

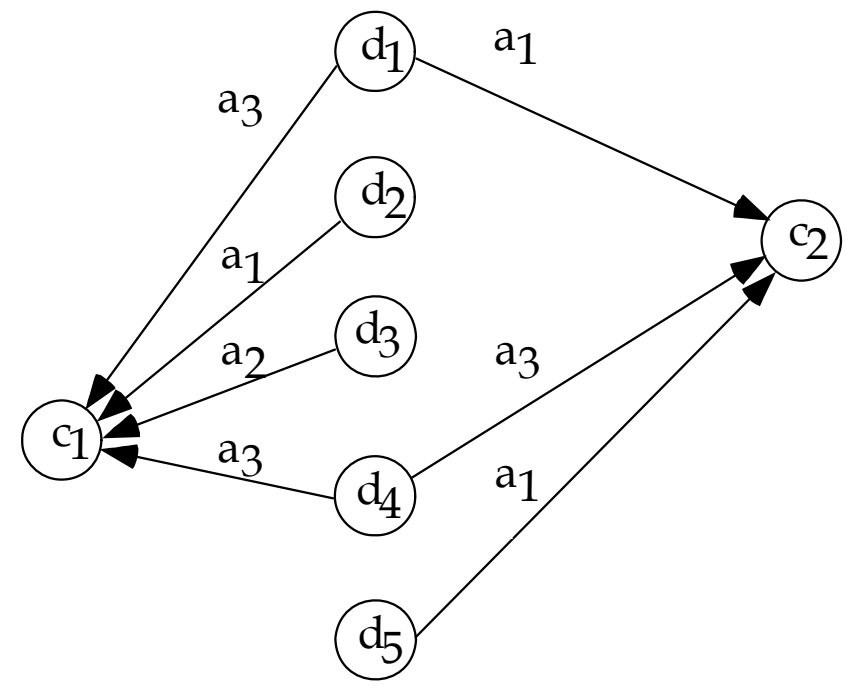

Figure 1: Marketing Network $G^{0}$

Observe, for example, that in marketing network $G^{0}$ both firms $d_{1}$ and $d_{5}$ offer product line $a_{1}$ in the Paris market (i.e., in the $c_{2}$ market). While all three product lines are offered in the New York market, only two product lines, $a_{1}$ and $a_{3}$, are offered in the Paris market, and only firm $d_{4}$ offers product line $a_{3}$ in the Paris market. Also note that all product line offerings are feasible (see the list above). Finally, note that four firms are active in the New York market; that is

$$
G^{0}\left(c_{1}\right):=\cup_{a \in A} G^{0}\left(a, c_{1}\right)=\left\{d_{1}, d_{2}, d_{3}, d_{4}\right\},
$$

while only three firms are active in the Paris market, that is,

$$
G^{0}\left(c_{2}\right):=\cup_{a \in A} G^{0}\left(a, c_{2}\right)=\left\{d_{1}, d_{4}, d_{5}\right\} .
$$

We shall maintain the following assumption throughout:

(A-1) (noncooperative free mobility) Each player can move freely and unilaterally from one club to another and each player can choose freely and unilaterally his feasible activity within the club.

Thus any player can drop his membership and activity in any given club and join any other club and take any other feasible action without bargaining with or seeking the permission of any player or group of players. In this sense, our model of club formation is noncooperative. The assumption of noncooperative free mobility is quite common in other models of noncooperative network formation (see, for example, Bala and Goyal 2000).

Example 2 Figure 2 depicts the marketing network which results when firm $d_{1}$ noncooperatively changes its product line-market profile from

$$
G^{0}\left(d_{1}\right)=\left\{\left(a_{3}, c_{1}\right),\left(a_{1}, c_{2}\right)\right\} \quad \text { to } G^{1}\left(d_{1}\right)=\left\{\left(a_{3}, c_{1}\right),\left(a_{1}, c_{2}\right),\left(a_{3}, c_{1}\right)\right\} .
$$




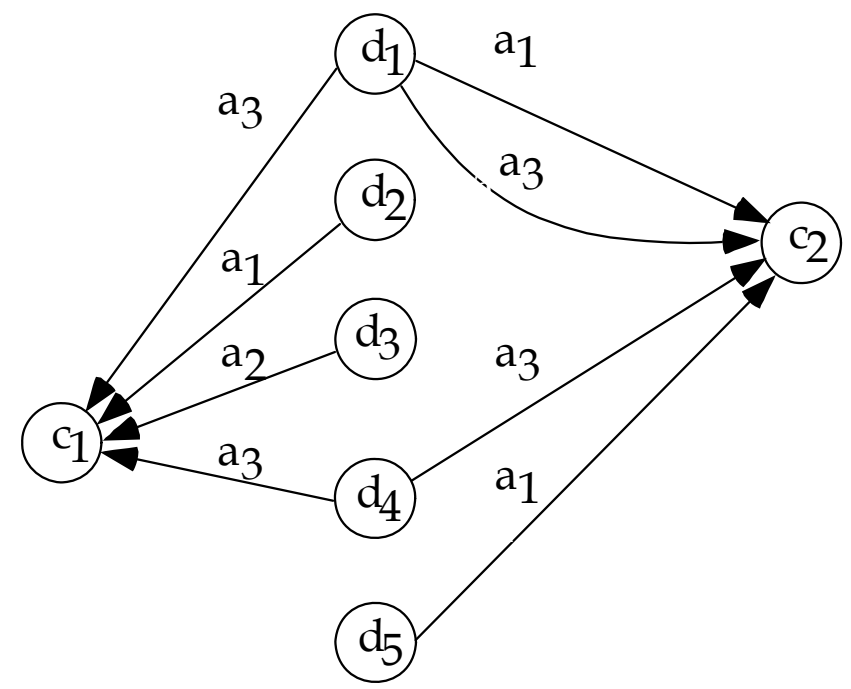

Figure 2: Marketing Network $G^{1}$

By thinking of marketing network $G^{0}$ as the 5-tuple of player networks,

$$
\left(G_{d_{1}}^{0}, G_{d_{2}}^{0}, \ldots, G_{d_{5}}^{0}\right)
$$

such that $G^{0}=\cup_{d_{i}} G_{d_{i}}^{0}$, we can represent the noncooperative move by firm 1 in the usual way as

$$
\left(G_{d_{1}}^{0}, G_{d_{2}}^{0}, \ldots, G_{d_{5}}^{0}\right)=\left(G_{d_{1}}^{0}, G_{-d_{1}}^{0}\right) \rightarrow\left(G_{d_{1}}^{1}, G_{-d_{1}}^{0}\right)=\left(G_{d_{1}}^{1}, G_{d_{2}}^{0}, \ldots, G_{d_{5}}^{0}\right)
$$

where

$$
\begin{gathered}
G_{d_{1}}^{0}=\left\{\left(a_{1},\left(d_{1}, c_{2}\right)\right),\left(a_{3},\left(d_{1}, c_{1}\right)\right)\right\} \in \mathbb{K}_{d_{1}} \\
\text { and } \\
G_{d_{1}}^{1}=\left\{\left(a_{1},\left(d_{1}, c_{2}\right)\right),\left(a_{3},\left(d_{1}, c_{1}\right)\right),\left(a_{3},\left(d_{1}, c_{2}\right)\right)\right\} \in \mathbb{K}_{d_{1}},
\end{gathered}
$$

and where

$$
G^{1}=G_{d_{1}}^{1} \cup\left(\cup_{d_{i} \neq d_{1}} G_{d_{i}}^{0}\right) .
$$

Thus, we can think of the move from $G^{0}$ to $G^{1}$ as being brought about by firm $d_{1}^{\prime} s$ noncooperatively changing its strategy from $G_{d_{1}}^{0}$ in strategy set $\mathbb{K}_{d_{1}}$ to $G_{d_{1}}^{1}$ in strategy set $\mathbb{K}_{d_{1}}$.

\section{Noncooperative Club Network Formation Games}

We will assume that (i) each players payoffs are additively separable in player specific payoffs, internal effects, and external effects; and (ii) that internal effects and external effects are homogenous across players. In particular, we will maintain the following assumption throughout: 
(A-2) (additive separability and externality homogeneity) Each player's real-valued payoff function $v_{d}(\cdot)$ defined on the set club networks $\mathbb{K}$ is given by

$$
v_{d}(G)=\sum_{(a, c) \in G(d)} r_{d}(a, c)+\sum_{(a, c) \in G(d)} I_{(a, c)}(|G(a, c)|)+\sum_{(a, c) \in G(d)^{c}} E_{(a, c)}(|G(a, c)|),
$$

where,

$G(d)^{c}$ is the complement of the set $G(d)$ in $A \times C$,

$r_{d}(a, c)$ is the player-specific payoff generated by the action-club pair, $(a, c) \in G(d)$, chosen by player $d$ in network $G$,

$I_{(a, c)}(|G(a, c)|)$ is the payoff externality generated by the number of players who choose the action-club pair, $(a, c)$, chosen by player $d$ in network $G$,

$\left.E_{(a, c)}(\mid G(a, c)) \mid\right)$ is the payoff externality generated by the number of players who choose an action-club pair $(a, c)$ in network $G$ not contained in the set of actionclub pairs $G(d)$ chosen by player $d$ in network $G$.

Note that for each action-club pair $(a, c)$, the functions $I_{(a, c)}(\cdot)$ and $E_{(a, c)}(\cdot)$ are the same for all players. The internal effect $I_{(a, c)}(|G(a, c)|)$ accrues to a player $d$ if and only if $(a, c)$ is contained in the set of action-club pairs chosen by player $d$ in network $G$; that is, if and only if $(a, c) \in G(d)$, whereas the external effect $E_{(a, c)}(|G(a, c)|)$ accrues to a player $d$ if and only if $(a, c)$ is not contained in the set of action-club pairs chosen by player $d$ in network $G$; that is, if and only if $(a, c) \notin G(d)$. Our specification of player payoffs given in (3) is a network rendition of a specification introduced in Hollard (2000).

Definitions 2 (Club Network Formation Games and Nash Equilibrium)

(a) A noncooperative club network formation game is specified by a D-tuple of pairs

$$
\left(\mathbb{K}_{d}, u_{d}(\cdot)\right)_{d \in D}
$$

where

(i) $\mathbb{K}_{d}$ is player d's strategy set (see Definition 1 (b)), and

(ii) $u_{d}(\cdot)$ is player d's real-valued payoff function defined on $\prod_{d} \mathbb{K}_{d}$ and specified as follows: for all $\left(G_{d}, G_{-d}\right) \in \prod_{d} \mathbb{K}_{d}$,

$$
u_{d}\left(G_{d}, G_{-d}\right):=v_{d}(G)
$$

where $G=\cup_{d} G_{d}$ and $v_{d}(G)$ is given by expression (3).

(b) A club network $G^{*}=\cup_{d} G_{d}^{*}$ is a Nash club network if for all players $d \in D$

$$
u_{d}\left(G_{d}^{*}, G_{-d}^{*}\right) \geq u_{d}\left(G_{d}, G_{-d}^{*}\right) \text { for all } G_{d} \in \mathbb{K}_{d}
$$




\section{Potentials and Nash Club Networks}

Our objective in this section is to show that under the assumptions of noncooperative free mobility (A-1) and additive separability and externality homogeneity (A-2), the club network formation game, $\left(\mathbb{K}_{d}, u_{d}(\cdot)\right)_{d \in D}$, with multiple club memberships is a finite potential game. This will allow us to conclude, in a manner similar to Monderer and Shapley (1996), that under assumptions (A-1) and (A-2) all noncooperative club network formation games with multiple memberships possess Nash club equilibria.

We begin by defining the notion of a potential game over club networks.

Definition 3 (Potential Games and Noncooperative Club Network Formation)

The noncooperative club network formation game $\left(\mathbb{K}_{d}, u_{d}(\cdot)\right)_{d \in D}$ is an exact potential game if there exists a real-valued function $P(\cdot)$ defined on the set of club networks $\mathbb{K}$ such that for all noncooperative changes

$$
\left(G_{d}^{0}, G_{-d}^{0}\right) \rightarrow\left(G_{d}^{1}, G_{-d}^{0}\right)
$$

brought about by any player $d \in D$

$$
u_{d}\left(G_{d}^{1}, G_{-d}^{0}\right)-u_{d}\left(G_{d}^{0}, G_{-d}^{0}\right)=P\left(G^{1}\right)-P\left(G^{0}\right) .
$$

where

$$
\begin{gathered}
G^{0}=\cup_{d} G_{d}^{0} \text { and } G^{1}=G_{d}^{1} \cup\left(\cup_{d^{\prime} \neq d} G_{d^{\prime}}^{0}\right) \\
\text { and } \\
u_{d}\left(G_{d}^{1}, G_{-d}^{0}\right)-u_{d}\left(G_{d}^{0}, G_{-d}^{0}\right):=v_{d}\left(G^{1}\right)-v_{d}\left(G^{0}\right) .
\end{gathered}
$$

We shall refer to any function satisfying equation (4) for all noncooperative changes as an exact potential function or as a potential.

It is easy to see that if $\left(\mathbb{K}_{d}, u_{d}(\cdot)\right)_{d \in D}$ is an exact potential game with potential $P(\cdot)$, then any club network contained in $\arg \max _{G \in \mathbb{K}} P(G)$ is a Nash club network for $\left(\mathbb{K}_{d}, u_{d}(\cdot)\right)_{d \in D}$. Moreover, since $\mathbb{K}$ is finite, $\arg \max _{G \in \mathbb{K}} P(G)$ is nonempty. Thus, one way to resolve the Nash problem for club network formation games is to show that these games possess potential functions. Our next objective, therefore, is to show that for club network formation games satisfying noncooperative free mobility (A-1) and additive separability and externality homogeneity (A-2) an exact potential function can be constructed.

Following Hollard (2000), let

$$
\Phi_{(a, c)}(k)=I_{(a, c)}(k)-E_{(a, c)}(k-1), k=0,1, \ldots,|D| .
$$

In club network $G \in \mathbb{K}$, if player $d$ chooses action-club pairs $G(d)$ and $(a, c) \in G(d)$, then

$$
\Phi_{(a, c)}(|G(a, c)|)=I_{(a, c)}(|G(a, c)|)-E_{(a, c)}(|G(a, c)|-1)
$$


is the difference between the internal effect derived by player $d$ in network $G$ from being in the group $G(a, c)$ taking action $a$ in club $c$ and the external effect player $d$ would derive from group $G(a, c)$ if player $d$ were to leave that group.

Our main result is the following:

Theorem (Club network formation games are potential games).

Let $\left(\mathbb{K}_{d}, u_{d}(\cdot)\right)_{d \in D}$ be a club network formation game satisfying noncooperative free mobility (A-1), payoff separability, and externality homogeneity (A-2). Then the function $P(\cdot): \mathbb{K} \rightarrow R$ given by

$$
P(G)=\sum_{(a, c) \in A \times C}\left[\sum_{d \in G(a, c)} r_{d}(a, c)+\sum_{k=0}^{|G(a, c)|} \Phi_{(a, c)}(k)\right],
$$

is an exact potential function for this game.

Since the proof consists primarily of long and tedious elementary algebra it is relegated to the appendix.

The following result is an immediate corollary of Theorem 1 above and Corollary 2.2 in Monderer and Shapley (1996).

Corollary (Club network formation games possess Nash club networks).

Let $\left(\mathbb{K}_{d}, u_{d}(\cdot)\right)_{d \in D}$ be a club network formation game satisfying noncooperative free mobility (A-1), payoff separability, and externality homogeneity (A-2). Then the set of Nash club networks $\mathbb{N} \mathbb{C N}$ is nonempty and for any potential, and in particular, for the potential given in expression (5),

$$
\arg \max _{G \in \mathbb{K}} P(G) \subseteq \mathbb{N} \mathbb{C} \text {. }
$$

In fact the argmax set corresponding to any weighted or any ordinal potential is also contained in the set of Nash club networks (see for example Monderer and Shapley (1996) for definitions and results).

Note that if we replace the set of clubs $C$ with the set of players $D$ in the club network formation above, then under assumptions (A-1) and (A-2) the resulting network formation game is still a potential game. In such a game, the typical network $G$ is a nonempty subset of the Cartesian product $A \times(D \times D)$ with typical element $\left(a,\left(d, d^{\prime}\right)\right.$, where $\left(a,\left(d, d^{\prime}\right)\right) \in G$ means that player $d$ initiates a feasible (or socially acceptable) action $a \in A\left(d, d^{\prime}\right)$ towards player $d^{\prime}$. As in the case of club networks, these networks - which we might call social interaction networks - can be represented as the union of player networks $G_{d} \subset A \times(\{d\} \times D)$, where each player network $G_{d}$ represents a player's social interaction strategy, and in particular, represents the actions a player directs toward the other players in network $G=\cup_{d} G_{d}$. 


\section{Consequences and Conclusions}

\subsection{The Kalai-Schmeidler Admissible Set, Basins of Attraction, and The Noncooperative Path Dominance Core}

Because all club network formation games satisfying noncooperative free mobility (A-1) and additive separability and externality homogeneity (A-2) are potential games, much more can be said about stability with respect to noncooperative network changes. In particular, we can conclude that no noncooperative improvement path forms a circuit and that each club network in $\mathbb{K}$ is either a Nash club network or is a network on a finite, noncooperative improvement path leading to a Nash club network (e.g., see section 3.3.3 and Theorem 1 in Page and Wooders, 2008). Thus, all club network formation games satisfying (A-1) and (A-2) have singleton basins of attraction (i.e., basins containing only one network), and thus all such games have unique, nonempty noncooperative path dominance cores (see Page and Wooders, 2008, Theorem 4). Moreover, in all club network formation games satisfying (A-1) and (A-2), the path dominance core is equal to the Kalai-Schmeidler admissible set (Kalai and Schmeidler, 1977) which is in turn equal to the set of Nash club networks.

\subsection{The Ui Shapley Value Representation}

Having done the tedious work of showing that club network formation games satisfying noncooperative free mobility (A-1) and having Hollard-type player payoff functions (A-2) are potential games, we can then use a remarkable Theorem due to Ui (2000) to conclude that, in fact, player payoff functions can be restated in terms of network-dependent Shapley values. In particular, by Theorem 2 in Ui (2000), for club network formation game $\left(\mathbb{K}_{d}, u_{d}(\cdot)\right)_{d \in D}$ satisfying (A-1) and (A-2) there exists a collection of network-dependent TU games $\left\{v_{G}(\cdot)\right\}_{G \in \mathbb{K}}$ such that for all club networks $G \in \mathbb{K}$

$$
u_{d}(G)=\phi_{d}\left(v_{G}(\cdot)\right):=\sum_{S \in \mathcal{N}, d \in S} \frac{(|S|-1) !(|D|-|S|) !}{|D| !}\left(v_{G}(S)-v_{G}(S \backslash\{d\})\right)
$$

where $u_{d}(\cdot)$ is player $d$ 's payoff function given in expression $(3), \mathcal{N}$ is the collection of all subsets of player set $D$, and $v_{G}(\cdot): \mathcal{N} \rightarrow R$ with $v_{G}(\emptyset)=0$ is a network-dependent TU game in characteristic function form.

\subsection{Other Relationships to the Literature}

Relative to the applications of the admissible set concept in Kalai and Schmeidler (1977), our model is restrictive in that both the number of arcs and nodes are finite. Much of the depth and beauty in the Kalai-Schmeidler results is in their treatment of situations with continuous strategy spaces and payoff sets. Fortunately, the KalaiSchmeidler methods can all be applied to infinite networks, as we demonstrate in research in progress.

The literature on economies with local public goods or clubs most closely related to the current paper is the line of literature including, for example, Demange (1994, 
2005) and Konishi, Le Breton and Weber (1997, 1998), who study economies with a fixed number of jurisdictions and free mobility of agents between jurisdictions.

In the literature on potential games, as we have already noted our results are related to those of Hollard (2000). Other important references in this literature include Rosenthal (1973), Myerson (1977), Hart and Mas-Colell (1989), Slade (1994), Monderer and Shapley (1996), Facchini, van Megen, Borm, and Tijs (1997), Ui (2000), Slikker, Dutta, van den Nouweland, and Tijs (2000), Voorneveld (2000), Slikker and van den Nouweland (2002), and Dubey, Haimanko, and Zapechelnyuk (2006).

\section{Appendix}

PROOF of the Theorem: Let $\left(G_{d_{1}}^{0}, G_{-d_{1}}^{0}\right) \rightarrow_{d_{1}}\left(G_{d_{1}}^{1}, G_{-d_{1}}^{0}\right)$ be a noncooperative network change brought about by player $d_{1} \in D$. We have

$$
\left.\begin{array}{l}
u_{d_{1}}\left(G_{d_{1}}^{1}, G_{-d_{1}}^{0}\right)-u_{d_{1}}\left(G_{d_{1}}^{0}, G_{-d_{1}}^{0}\right) \\
=\left(\sum_{(a, c) \in G^{1}\left(d_{1}\right)} r_{d_{1}}(a, c)-\sum_{(a, c) \in G^{0}\left(d_{1}\right)} r_{d_{1}}(a, c)\right) \\
+\left(\sum_{(a, c) \in G^{1}\left(d_{1}\right)} I_{(a, c)}\left(\left|G^{1}(a, c)\right|\right)-\sum_{(a, c) \in G^{0}\left(d_{1}\right)} I_{(a, c)}\left(\left|G^{0}(a, c)\right|\right)\right) \\
+\left(\sum_{(a, c) \in G^{1}\left(d_{1}\right)^{c}} E_{(a, c)}\left(\left|G^{1}(a, c)\right|\right)-\sum_{(a, c) \in G^{0}\left(d_{1}\right)^{c}} E_{(a, c)}\left(\left|G^{0}(a, c)\right|\right)\right) .
\end{array}\right\}
$$

First, observe that,

$$
\left.\begin{array}{l}
\sum_{(a, c) \in G^{1}\left(d_{1}\right)} r_{d_{1}}(a, c)-\sum_{(a, c) \in G^{0}\left(d_{1}\right)} r_{d_{1}}(a, c) \\
=\sum_{(a, c) \in G^{1}\left(d_{1}\right) \backslash G^{0}\left(d_{1}\right)} r_{d_{1}}(a, c)-\sum_{(a, c) \in G^{0}\left(d_{1}\right) \backslash G^{1}\left(d_{1}\right)} r_{d_{1}}(a, c) .
\end{array}\right\}
$$

Second, observe that

$$
\left.\begin{array}{c}
\text { for all }(a, c) \in\left(G^{1}\left(d_{1}\right) \cap G^{0}\left(d_{1}\right)\right) \cup\left(A \times C \backslash\left(G^{1}\left(d_{1}\right) \cup G^{0}\left(d_{1}\right)\right),\right. \\
\qquad\left|G^{1}(a, c)\right|=\left|G^{0}(a, c)\right| .
\end{array}\right\}
$$

Thus,

$$
\begin{aligned}
& \sum_{(a, c) \in G^{1}\left(d_{1}\right)} I_{(a, c)}\left(\left|G^{1}(a, c)\right|\right)-\sum_{(a, c) \in G^{0}\left(d_{1}\right)} I_{(a, c)}\left(\left|G^{0}(a, c)\right|\right) \\
& =\sum_{(a, c) \in G^{1}\left(d_{1}\right) \backslash G^{0}\left(d_{1}\right)} I_{(a, c)}\left(\left|G^{1}(a, c)\right|\right) \\
& -\sum_{(a, c) \in G^{0}\left(d_{1}\right) \backslash G^{1}\left(d_{1}\right)} I_{(a, c)}\left(\left|G^{0}(a, c)\right|\right)
\end{aligned}
$$


and

$$
\begin{aligned}
& \sum_{(a, c) \in G^{1}\left(d_{1}\right)^{c}} E_{(a, c)}\left(\left|G^{1}(a, c)\right|\right)-\sum_{(a, c) \in G^{0}\left(d_{1}\right)^{c}} E_{(a, c)}\left(\left|G^{0}(a, c)\right|\right) \\
& =\sum_{(a, c) \in G^{0}\left(d_{1}\right) \backslash G^{1}\left(d_{1}\right)} E_{(a, c)}\left(\left|G^{1}(a, c)\right|\right) \\
& -\sum_{(a, c) \in G^{1}\left(d_{1}\right) \backslash G^{0}\left(d_{1}\right)} E_{(a, c)}\left(\left|G^{0}(a, c)\right|\right) \\
& =\sum_{(a, c) \in G^{0}\left(d_{1}\right) \backslash G^{1}\left(d_{1}\right)} E_{(a, c)}\left(\left|G^{0}(a, c)\right|-1\right) \\
& -\sum_{(a, c) \in G^{1}\left(d_{1}\right) \backslash G^{0}\left(d_{1}\right)} E_{(a, c)}\left(\left|G^{1}(a, c)\right|-1\right) .
\end{aligned}
$$

From (8)-(11) we conclude therefore that

$$
\begin{aligned}
& u_{d_{1}}\left(G_{d_{1}}^{1}, G_{-d_{1}}^{0}\right)-u_{d_{1}}\left(G_{d_{1}}^{0}, G_{-d_{1}}^{0}\right) \\
& =\sum_{(a, c) \in G^{1}\left(d_{1}\right) \backslash G^{0}\left(d_{1}\right)} r_{d_{1}}(a, c) \\
& +\left[\sum_{(a, c) \in G^{1}\left(d_{1}\right) \backslash G^{0}\left(d_{1}\right)}\left(I_{(a, c)}\left(\left|G^{1}(a, c)\right|\right)-E_{(a, c)}\left(\left|G^{1}(a, c)\right|-1\right)\right)\right] \\
& -\sum_{(a, c) \in G^{0}\left(d_{1}\right) \backslash G^{1}\left(d_{1}\right)} r_{d_{1}}(a, c) \\
& -\left[\sum_{(a, c) \in G^{0}\left(d_{1}\right) \backslash G^{1}\left(d_{1}\right)}\left(I_{(a, c)}\left(\left|G^{0}(a, c)\right|\right)-E_{(a, c)}\left(\left|G^{0}(a, c)\right|-1\right)\right)\right] \\
& =\sum_{(a, c) \in G^{1}\left(d_{1}\right) \backslash G^{0}\left(d_{1}\right)}\left[r_{d_{1}}(a, c)+\Phi_{(a, c)}\left(\left|G^{1}(a, c)\right|\right)\right] \\
& -\left(\sum_{(a, c) \in G^{0}\left(d_{1}\right) \backslash G^{1}\left(d_{1}\right)}\left[r_{d_{1}}(a, c)+\Phi_{(a, c)}\left(\left|G^{0}(a, c)\right|\right)\right]\right) .
\end{aligned}
$$

Next consider $P\left(G^{1}\right)-P\left(G^{0}\right)$. We have,

$$
\left.\begin{array}{l}
P\left(G^{1}\right)-P\left(G^{0}\right) \\
=\sum_{(a, c)}\left[\sum_{d \in G^{1}(a, c)} r_{d}(a, c)+\sum_{k=0}^{\left|G^{1}(a, c)\right|} \Phi_{(a, c)}(k)\right] \\
-\sum_{(a, c)}\left[\sum_{d \in G^{0}(a, c)} r_{d}(a, c)+\sum_{k=0}^{\left|G^{0}(a, c)\right|} \Phi_{(a, c)}(k)\right] \cdot
\end{array}\right\}
$$

For $G \in \mathbb{K}$ let

$$
H(G):=\{(a, c) \in A \times C:(a,(d, c)) \in G \text { for some } d \in D\},
$$

and note that

$$
H\left(G^{1}\right) \backslash\left[G^{1}\left(d_{1}\right) \cup G^{0}\left(d_{1}\right)\right]=H\left(G^{0}\right) \backslash\left[G^{1}\left(d_{1}\right) \cup G^{0}\left(d_{1}\right)\right] .
$$

Letting

$$
S:=\left[H\left(G^{1}\right) \backslash G^{1}\left(d_{1}\right)\right] \cup G^{0}\left(d_{1}\right)=\left[H\left(G^{0}\right) \backslash G^{1}\left(d_{1}\right)\right] \cup G^{0}\left(d_{1}\right),
$$


now observe that

$$
\begin{aligned}
& \sum_{(a, c)} \sum_{d \in G^{1}(a, c)} r_{d}(a, c)-\sum_{(a, c)} \sum_{d \in G^{0}(a, c)} r_{d}(a, c) \\
& =\sum_{(a, c) \in S \cup\left(G^{1}\left(d_{1}\right) \cap G^{0}\left(d_{1}\right)\right)}\left(\sum_{d \in G^{1}(a, c)} r_{d}(a, c)-\sum_{d \in G^{0}(a, c)} r_{d}(a, c)\right) \\
& +\sum_{(a, c) \in G^{1}\left(d_{1}\right) \backslash G^{0}\left(d_{1}\right)}\left(\sum_{d \in G^{1}(a, c)} r_{d}(a, c)-\sum_{d \in G^{0}(a, c)} r_{d}(a, c)\right) \\
& +\sum_{(a, c) \in G^{0}\left(d_{1}\right) \backslash G^{1}\left(d_{1}\right)}\left(\sum_{d \in G^{1}(a, c)} r_{d}(a, c)-\sum_{d \in G^{0}(a, c)} r_{d}(a, c)\right) .
\end{aligned}
$$

Moreover, note that

$$
\begin{gathered}
\sum_{(a, c) \in S \cup\left(G^{1}\left(d_{1}\right) \cap G^{0}\left(d_{1}\right)\right)}\left(\sum_{d \in G^{1}(a, c)} r_{d}(a, c)-\sum_{d \in G^{0}(a, c)} r_{d}(a, c)\right)=0 \\
\sum_{(a, c) \in G^{1}\left(d_{1}\right) \backslash G^{0}\left(d_{1}\right)}\left(\sum_{d \in G^{1}(a, c)} r_{d}(a, c)-\sum_{d \in G^{0}(a, c)} r_{d}(a, c)\right) \\
=\sum_{(a, c) \in G^{1}\left(d_{1}\right) \backslash G^{0}\left(d_{1}\right)} r_{d_{1}}(a, c) \\
\text { and } \\
\sum_{(a, c) \in G^{0}\left(d_{1}\right) \backslash G^{1}\left(d_{1}\right)}\left(\sum_{d \in G^{1}(a, c)} r_{d}(a, c)-\sum_{d \in G^{0}(a, c)} r_{d}(a, c)\right) \\
=-\sum_{(a, c) \in G^{0}\left(d_{1}\right) \backslash G^{1}\left(d_{1}\right)} r_{d_{1}}(a, c) .
\end{gathered}
$$

Therefore,

$$
\left.\begin{array}{c}
\sum_{(a, c)} \sum_{d \in G^{1}(a, c)} r_{d}(a, c)-\sum_{(a, c)} \sum_{d \in G^{0}(a, c)} r_{d}(a, c) \\
\sum_{(a, c) \in G^{1}\left(d_{1}\right) \backslash G^{0}\left(d_{1}\right)} r_{d_{1}}(a, c)-\sum_{(a, c) \in G^{0}\left(d_{1}\right) \backslash G^{1}\left(d_{1}\right)} r_{d_{1}}(a, c) .
\end{array}\right\}
$$

Next, observe that

$$
\begin{gathered}
\sum_{(a, c)} \sum_{k=0}^{\left|G^{1}(a, c)\right|} \Phi_{(a, c)}(k)-\sum_{(a, c)} \sum_{k=0}^{\left|G^{0}(a, c)\right|} \Phi_{(a, c)}(k) \\
=\sum_{(a, c) \in S \cup\left(G^{1}\left(d_{1}\right) \cap G^{0}\left(d_{1}\right)\right)}\left(\sum_{k=0}^{\left|G^{1}(a, c)\right|} \Phi_{(a, c)}(k)-\sum_{k=0}^{\left|G^{0}(a, c)\right|} \Phi_{(a, c)}(k)\right) \\
+\sum_{(a, c) \in G^{1}\left(d_{1}\right) \backslash G^{0}\left(d_{1}\right)} \Phi_{(a, c)}\left(\left|G^{1}(a, c)\right|\right) \\
-\sum_{(a, c) \in G^{0}\left(d_{1}\right) \backslash G^{1}\left(d_{1}\right)} \Phi_{(a, c)}\left(\left|G^{0}(a, c)\right|\right) .
\end{gathered}
$$

Moreover, given the definition of the set $S$,

$$
\sum_{(a, c) \in S \cup\left(G^{1}\left(d_{1}\right) \cap G^{0}\left(d_{1}\right)\right)}\left(\sum_{k=0}^{\left|G^{1}(a, c)\right|} \Phi_{(a, c)}(k)-\sum_{k=0}^{\left|G^{0}(a, c)\right|} \Phi_{(a, c)}(k)\right)=0 .
$$


From (14)-(18) we conclude that

$$
\begin{aligned}
& P\left(G^{1}\right)-P\left(G^{0}\right) \\
& =\sum_{(a, c) \in G^{1}\left(d_{1}\right) \backslash G^{0}\left(d_{1}\right)} r_{d_{1}}(a, c)+\sum_{(a, c) \in G^{1}\left(d_{1}\right) \backslash G^{0}\left(d_{1}\right)} \Phi_{(a, c)}\left(\left|G^{1}(a, c)\right|\right) \\
& -\sum_{(a, c) \in G^{0}\left(d_{1}\right) \backslash G^{1}\left(d_{1}\right)} r_{d_{1}}(a, c)-\sum_{(a, c) \in G^{0}\left(d_{1}\right) \backslash G^{1}\left(d_{1}\right)} \Phi_{(a, c)}\left(\left|G^{0}(a, c)\right|\right) \\
& =\sum_{(a, c) \in G^{1}\left(d_{1}\right) \backslash G^{0}\left(d_{1}\right)}\left[r_{d_{1}}(a, c)+\Phi_{(a, c)}\left(\left|G^{1}(a, c)\right|\right)\right] \\
& -\left(\sum_{(a, c) \in G^{0}\left(d_{1}\right) \backslash G^{1}\left(d_{1}\right)}\left[r_{d_{1}}(a, c)+\Phi_{(a, c)}\left(\left|G^{0}(a, c)\right|\right)\right]\right) .
\end{aligned}
$$

Thus, we conclude that for all noncooperative changes, $\left(G_{d}^{0}, G_{-d}^{0}\right) \rightarrow\left(G_{d}^{1}, G_{-d}^{0}\right)$, brought about by any player $d \in D$,

$$
u_{d}\left(G_{d}^{1}, G_{-d}^{0}\right)-u_{d}\left(G_{d}^{0}, G_{-d}^{0}\right)=P\left(G^{1}\right)-P\left(G^{0}\right),
$$

where

$$
\begin{gathered}
u_{d_{1}}\left(G_{d}^{1}, G_{-d}^{0}\right)-u_{d}\left(G_{d}^{0}, G_{-d}^{0}\right):=v_{d}\left(G^{1}\right)-v_{d}\left(G^{0}\right), \\
\quad \text { and } \\
G^{0}=\cup_{d^{\prime} \in D} G_{d^{\prime}}^{0} \text { and } G^{1}=G_{d}^{1} \cup\left(\cup_{d^{\prime} \neq d} G_{d^{\prime}}^{0}\right) .
\end{gathered}
$$

\section{References}

[1] Allouch, N. and M. Wooders (2007) "Price taking equilibrium in economies with multiple memberships in clubs and unbounded club sizes," Journal of Economic Theory (to appear).

[2] Bala, V. and S. Goyal (2000) "A noncooperative model of network formation," Econometrica 68, 1181-1229.

[3] Buchanan, J. (1965) "An economic theory of clubs," Economica 33, 1-14.

[4] Demange, G. (2004) "On group stability and hierarchies in networks," Journal of Political Economy 112, 754-778.

[5] Demange G. (1994) "Intermediate preferences and stable coalition structures," Journal of Mathematical Economics, 1994, 45-48.

[6] Dubey, P., O. Haimanko, and A. Zapechelnyuk (2006) "Strategic complements and substitutes, and potential games," Games and Economic Behavior 54, 77-94.

[7] Facchini, G., F. van Megen, P. Born, and S. Tijs (1997) "Congestion games and weighted Bayesian potential games," Theory and Decision 42, 193-206.

[8] Hart, S. and A. Mas-Colell (1989) "Potential, Value, and Consistency," Econometrica 57, 589-614. 
[9] Hollard, Guillaume (2000) "On the existence of a pure strategy Nash equilibrium in group formation games," Economics Letters 66, 283-287.

[10] Kalai, E., A. Pazner, and D. Schmeidler (1976) "Collective choice correspondences as admissible outcomes of social bargaining processes," Econometrica 44, 233-240.

[11] Kalai, E.,and D. Schmeidler (1977) "An admissible set occurring in various bargaining situations," Journal of Economic Theory 14, 402-411.

[12] Konishi, H., M. Le Breton, and S. Weber (1997): "Free mobility equilibrium in a local public goods economy with congestion," Research in Economics 51, 19-30.

[13] Konishi, H., M. Le Breton, and S. Weber (1998) "Equilibrium in a finite local public goods economy," Journal of Economic Theory 79, 224-244.

[14] Monderer, D., and L. Shapley (1996) "Potential games," Games and Economic Behavior 14, 124-143.

[15] Myerson, R.B. (1977) "Graphs and cooperation in games," Mathematics of Operations Research 2, 225-229.

[16] Page, Jr., F. H., and M. Wooders (2005) "Strategic basins of attraction, the farsighted core, and network formation games," FEEM Working Paper 2005.36.

[17] Page, Jr., F. H., and M. Wooders (2008) "Strategic basins of attraction, the path dominance core, and network formation games," in press Games and Economic Behavior, doi:10.1016/j.geb.2008.05.003.

[18] Rosenthal, R. W. (1973) "A class of games possessing pure-strategy Nash equilibria," International Journal of Game Theory 2, 65-67.

[19] Slade, M. E. (1994) "What does an oligopoly maximize," Journal of Industrial Economics 42, 45-61.

[20] Slikker, M., B. Dutta, A. van den Nouweland and S. Tijs (2000) "Potential maximizers and network formation," Mathematical Social Sciences 39, 55-70.

[21] Slikker, M. and A. van den Nouweland (2002) "Network formation, costs and potential games," in Chapters in Game Theory, P. Borm and H. Peters eds. Kluwer Academic Publishers, the Netherlands.

[22] Tiebout, C. (1956) "A pure theory of local expenditures," Journal of Political Economy 64, 416-424.

[23] Ui, T. (2000) "A Shapley value representation of potential games," Games and Economic Behavior 31, 121-135.

[24] Voorneveld, M. (2000) "Best-response potential games," Economics Letters 66, 289-295. 